\title{
Meshed Multipath Routing: An Efficient Strategy in Sensor Networks
}

\author{
Swades De ${ }^{1}$, Chunming Qiao ${ }^{2}$, and Hongyi $\mathrm{Wu}^{3}$ \\ ${ }^{1}$ Department of Electrical Engineering \\ ${ }^{2}$ Department of Computer Science and Engineering \\ ${ }^{1,2}$ State University of New York at Buffalo, Buffalo, NY 14260 \\ ${ }^{3}$ The Center for Advanced Computer Studies \\ ${ }^{3}$ University of Louisiana at Lafayette, Lafayette, LA 70504 \\ \{swadesd, qiao\}@cse.buffalo.edu, wulcacs.louisiana.edu
}

\begin{abstract}
Due to limited functionalities and potentially large number of sensors, conventional routing strategies proposed for distributed control applications (such as mobile ad hoc networks) are not directly applicable in wireless sensor networks. In this paper, we propose a novel meshed multipath routing (M-MPR) with selective forwarding of packets. Our evaluation shows that M-MPR achieves much improved throughput performance over conventional disjoint multipath routing, with comparable power consumption and receiver complexity. We also show that for comparable throughput, M-MPR achieves better load distribution and requires lesser route maintenance overhead with respect to packet forwarding along a preferred route.
\end{abstract}

\section{INTRODUCTION}

A wireless sensor network is similar to mobile ad hoc networks, but it differs from them in the sense that the nodes have much reduced functionalities, such as limited transmission range and battery power [1]. While re-transmission can be used to recover from data loss, basic sensors may not have enough storage space to save the collected information for necessary re-transmission. Moreover, some data require real-time or almost realtime communication (e.g., dynamically changing field images), thus disallowing re-transmission delay, even if the sensors are capable.

To facilitate immediate and successful data delivery, we resort to setting up meshed multiple routes from a source (e.g., a field sensor) to a destination (e.g., the clusterhead). This allows (some, if not all) nodes in the route to have more than one forwarding directions to the destination. Data transmission is done via selective forwarding of packets, where the routing decision is taken dynamically, hop-by-hop, based on the conditions of downstream forwarding channels. Forward error correction coding is used to avoid acknowledgment-based re-transmission.

Before proceeding further, we briefly survey the related work. For high-speed transmission in intercon- nection networks, virtual cut-through routing [7] and wormhole routing [2] have been implemented, where end-to-end acknowledgment is used to deal with link error and node failure. To avoid deadlock problem in wormhole routing, either deterministic routing or adaptive routing along with end-to-end handshaking is used. In mobile ad hoc networks and sensor networks, to ensure delay and/or loss guarantee, multiple disjoint [3] or partially disjoint [9],[5] routes are set up and data is transmitted along primary route while the unused secondary routes are maintained via periodic control signaling. To deal with network error, either end-to-end [3] or adjacent node [5] acknowledgment based rerouting is done. Traffic splitting along disjoint multiple routes [8] (called disjoint multipath routing, or D-MPR), aims at network load balancing. For a given channel error probability, [11] studied optimum number of disjoint multiple routes to ensure successful data delivery. In directed diffusion [6], a single-path route from sink to the source is set up, decided upon the interest gradient of data. Credit-based mesh forwarding [12] introduces flexibility of a single-path route selection in dynamic network conditions.

The distinct features of our meshed multipath routing (M-MPR) over the existing multipath approaches are the following: (a) Instead of splitting traffic along disjoint multipath [8],[11], meshed route introduces more flexibility in on-the-fly routing decision. (b) Instead of sending traffic along a preferential (primary) route among a number of disjoint or partially disjoint multiple alternatives [3],[9],[6],[5], M-MPR distributes traffic evenly in the mesh, thereby achieving better load balancing and requiring lesser signaling overhead for multiple route maintenance. (c) Unlike in [7],[2],[3],[5], the absence of acknowledgment-based re-transmission and rerouting simplifies the flow control mechanism, and reduces energy and buffer requirements at the field sensors. 
In this paper, we focus on throughput and load balancing performances of our proposed M-MPR scheme. Our evaluation shows that (i) in terms of throughput, MMPR outperforms its node equivalent as well as receiver complexity equivalent D-MPR; (ii) throughput gain of M-MPR is more for longer end-to-end distance; (iii) for comparable throughput performance, M-MPR distributes traffic more evenly along the meshed route and requires lesser route maintenance overhead compared to packet forwarding along the primary route.

In the following, the M-MPR scheme is introduced in Section II; Section III contains throughput analyses of M-MPR and D-MPR; simulation based verification of our analysis, and load balancing performance studies are conducted in Section IV; Section V concludes the paper.

\section{Meshed Multipath Routing}

In this section, firstly we outline the meshed route formation strategies. Then the selective packet forwarding is described.

\section{A. Meshed Multipath Searching}

In conventional disjoint route searching, an intermediate node entertains only one query packet. Since here a meshed route is intended, an intermediate node is allowed to receive (and record) more than one query packet. Typically, to limit the receiver complexity ${ }^{1}$ and power consumption, we restrict to maximum two query packets to be received and only one (first arrival) to be forwarded to maximum two downstream neighbors. In choosing the downstream nodes, our underlying assumption is that a node is aware of relative locations of its local neighbors. Since the sensors are mostly static, the nodes' location information can be imparted during their initial deployment phase via standard trilateration approach using other GPS-capable and possibly mobile nodes [4]. Note that a query packet is forwarded to only those neighbors from which no query has been received. If a node sends a query packet to a neighbor and then receives a query packet from the same neighbor (this is possible when the two nodes send a query packet to each at about the same time), no directed links between the two nodes will be formed as a part of the mesh. This enables formation of loop-less meshed routes. Once the destination receives the query packet(s), it replies to a subset of them with reservation confirmation packets, which can simply follow the directed links (though in opposite direction). We call this approach back-to-back (or single-ended) meshed route discovery (see Fig.1 (a) for an illustration).

\footnotetext{
${ }^{1}$ Receiver complexity of a node is a function of the number of incoming links.
}

We observe that in single-ended route searching, many of the nodes involved in the searching process do not take part in actual packet forwarding (see, for example, the nodes in Fig. 1 (a) represented by hollow circles). If the locations of source and destination are known to each other, or if suitable coordination between them can be achieved through a third party, the two nodes can initiate the multiple path searching process concurrently using an approach called double-ended route searching to further reduce the searching delay and control overhead (see Fig. 1 (b) for an illustration). Due to limited space, we omit the detailed description of various searching methods, but instead, concentrate on the performance evaluation of the proposed M-MPR scheme.

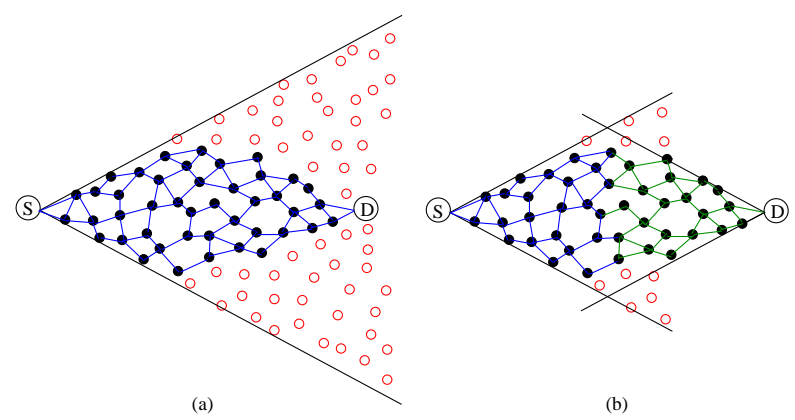

Fig. 1. Pictorial views of meshed point-to-point route searching schemes, where maximum degree of incoming and outgoing connectivities of a node are limited to two. (a) single-ended route search, (b) double-ended route search.

\section{B. Multipath Routing with Selective Forwarding}

Once the meshed route is constructed, along the meshed multipath at each node packets are forwarded to only one downstream outgoing link based on local conditions. If all outgoing links at a node are equally good, one is selected randomly. Besides fault tolerance, selective forwarding in M-MPR can distribute the traffic among multiple routes and hence achieve high speed transmission. Also, it can refresh a node's association with the mesh, thereby minimizing the need for explicit route maintenance. In case of channel error or node failure, the intermediate nodes are responsible for maintaining (or altering) their connectivities with the mesh.

It may be noted that node-to-node communication in wireless scenario, is generally broadcast-based (which is specifically true for simple field sensors). Therefore, meshed multipath formation is a more natural choice in sensor networks, without requiring any extra transmission power and channel resource (e.g., orthogonal codes) to reach more than one local neighbor. Also, since a node has only partial network (i.e., local neighborhood) information, meshed route introduces more flexibility in routing decision (compared to end-to-end route condition 
based end node controlled routing decision). However, this hop-by-hop routing decision (similar to adaptive routing) may lead to non-optimal route selection.

\section{Throughrut AnAlysis}

We now evaluate the throughput performances of MMPR and D-MPR schemes. The source-to-destination hop length is denoted by $H$, where all routes are assumed to be of equal length and the meshed multipath is mostly regular (see Figs. 2 and 3). Note that although the "equal length routes" and "regular mesh" assumptions may not be very practical, with these assumptions, the system lends itself to tractable analytic performance evaluation which can be used to gain intuitive understanding of routing performances. In section IV, we will consider more realistic cases via simulations.

With maximum two incoming or outgoing branches at a node, the number of nodes involved in M-MPR is:

$$
N^{(M)}= \begin{cases}\left(\frac{H+2}{2}\right)^{2}, & H \text { even } \\ \left\lceil\frac{H}{2}\right\rceil\left(\left\lceil\frac{H}{2}\right\rceil+1\right), & H \text { odd. }\end{cases}
$$

On the other hand, the number of nodes associated with $r$ disjoint $H$-hop source-destination routes in DMPR is:

$$
N^{(D)}=r(H-1)+2
$$

Hereafter, for each packet transmission, link error and intermediate node failure probabilities are denoted by $p_{l}$ and $p_{n}$, respectively. Note that the end node (i.e., the destination) is considered good (i.e., $p_{n}=0$ ) for all packets, as it is a primary entity (along with the source) in the communication process. $p_{l}$ captures multiuser interference caused by medium access conflict, and $p_{n}$ captures the packet loss due to input buffer overflow along with the node failure. A link is modeled as additive white Gaussian noise (AWGN) channel. If $p_{b}$ is the average bit error probability (or BER) due to channel error and $B$ is the packet size (number of bits), then

$$
p_{l}=1-\left(1-p_{b}\right)^{B}
$$

If direct sequence spread spectrum technique is used, with $K$ contending nodes and $C$ chips per bit, Gaussian approximation yields the average BER [10, p. 282]

$$
p_{b}=Q\left(\frac{1}{\sqrt{\frac{K-1}{3 C}+\frac{N_{0}}{2 E_{b}}}}\right)
$$

where $E_{b} / N_{0}$ is the signal-to-noise ratio per bit.

\section{A. Meshed Multipath}

There could be different ways of forming meshed multipath. To facilitate fair comparative analysis we consider the meshed routes as shown in Fig. 2. We denote the intermediate nodes by $N_{i j}$ where $i$ stands for
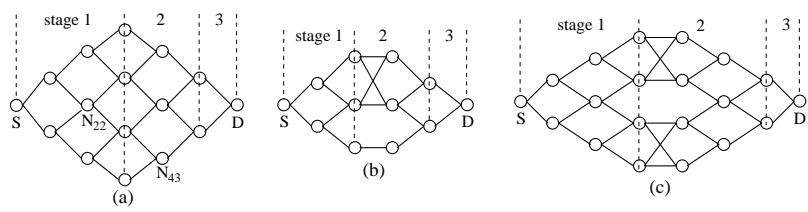

Fig. 2. Examples of meshed multipath (a) Even number of hops $(H=6)$; (b) odd number of hops $(H=5),\left\lfloor\frac{H}{2}\right\rfloor$ even; (c) odd number of hops $(H=7),\left\lfloor\frac{H}{2}\right\rfloor$ odd.

the hop length from source and $j$ stands for its position from top of the mesh.

Depending on the hop length, the meshed route is divided into three stages. Stage 1 covers the nodes from the source up to those $\left\lfloor\frac{H}{2}\right\rfloor$ hops away, Stage 2 covers the hop between $\left\lfloor\frac{H}{2}\right\rfloor$ and $H-1$, and Stage 3 is the last hop. Successful packet arrival probability at the end of each stage, denoted by $P_{s}(i)$, where $i=1,2$, is obtained as shown below:

Stage 1: In this stage, a packet successfully reaches the next node if at least one of two downstream nodes is good, with probability $\left(1-p_{n}^{2}\right)$, and the channel is good during the packet transmission, with probability $\left(1-p_{l}\right)$. Considering up to $\left\lfloor\frac{H}{2}\right\rfloor$ hops, $P_{s}(1)$ is given by

$$
P_{s}(1)=\left[\left(1-p_{l}\right)\left(1-p_{n}^{2}\right)\right]^{\left\lfloor\frac{H}{2}\right\rfloor} \text {. }
$$

The probability with which a successful packet arrives at a node $N_{i j}$ at the end of Stage 1 is binomially distributed:

$$
P_{h, i+1}=\frac{1}{2^{h}}\left(\begin{array}{c}
h \\
i
\end{array}\right)
$$

where $h=\left\lfloor\frac{H}{2}\right\rfloor$ and $i=0,1, \cdots, h$.

Stage 2: $P_{s}(2)$ is obtained recursively as shown in Appendix A. Note that the edge nodes beyond $\left\lceil\frac{H}{2}\right\rceil$ hops (e.g., $N_{43}$ in Fig. 2(a)) have only one downstream node.

Finally, counting the stage 3, end-to-end successful arrival probability of a packet, or normalized throughput is given by

$$
T_{S F}^{(M)}=\left(1-p_{l}\right) \prod_{i=1}^{2} P_{s}(i)
$$

\section{B. Disjoint Multipath}

An example of disjoint multipath is shown in Fig. 3.

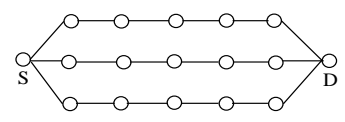

Fig. 3. Example of 6-hop disjoint multiple routes.

In D-MPR, routing decision flexibility is available only at the source. The corresponding normalized throughput is given by

$$
T^{(D)}=\left(1-p_{l}\right)^{H}\left(1-p_{n}^{r}\right)\left(1-p_{n}\right)^{H-2}
$$


where $\left(1-p_{l}\right)\left(1-p_{n}^{r}\right)$ is the probability of reaching to a next node from the source.

\section{Performance Comparison}

Numerical results for the above routing strategies are provided here.

Throughput Performance: To compare the throughput of M-MPR with its node equivalent D-MPR, we determine the number of disjoint routes, $r$ in D-MPR, so that $N^{(M)} \simeq N^{(D)}$ (Eqs. (1) and (2)). Throughput gain in M-MPR over its node-equivalent D-MPR is shown in Fig. 4, where increased vulnerability of disjoint MPR is apparent as the route gets longer. As a reason of poor performance of D-MPR, we note that once a route is decided at the source end, no further alternate routing option is available. Hence, any failure at the intermediate stage implies packet loss. On the other hand, in M-MPR this flexibility is extended throughout the route.

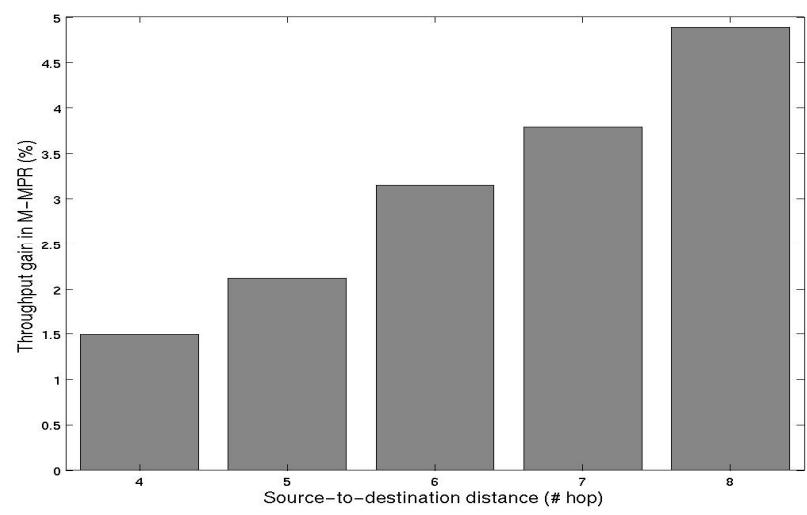

Fig. 4. Percentage throughput gain in M-MPR over its node equivalent D-MPR scheme. $p_{b}=10^{-6}, p_{n}=10^{-2}$.

Receiver Complexity: To compare the resource usage (i.e., number of orthogonal codes required and receiver complexity), without loss of generality we assume that the underlying MAC layer uses spread spectrum technique, where each node has its unique (orthogonal) code for transmission. We do not consider spatial separation dependent code reuse. Thus, the number of orthogonal codes required is equal to the number of transmitting nodes $(N)$ along the route, and the number of correlators required in a receiver is equal to the number of incoming links. Total number of correlators required $(R)$ in a multipath route determines the receiver complexity of the routing scheme.

Considering M-MPR and its node equivalent as well as receiver complexity equivalent D-MPR, the Fig. 5 shows the normalized throughput of different routing schemes for a 6-hop route. We note that M-MPR with equal number of nodes $\left(N^{(M)}=N^{(D)}=22\right)$, but with higher receiver complexity $\left(R^{(M)}=40\right.$ against $R^{(D)}=$
24), has higher throughput. With equal receiver complexity $\left(R^{(M)}=R^{(D)}=24\right)$, M-MPR still performs better over D-MPR. It may be noted that receiver complexity equivalent M-MPR involves lesser nodes $\left(N^{(M)}=16\right.$ against $N^{(D)}=22$ ).

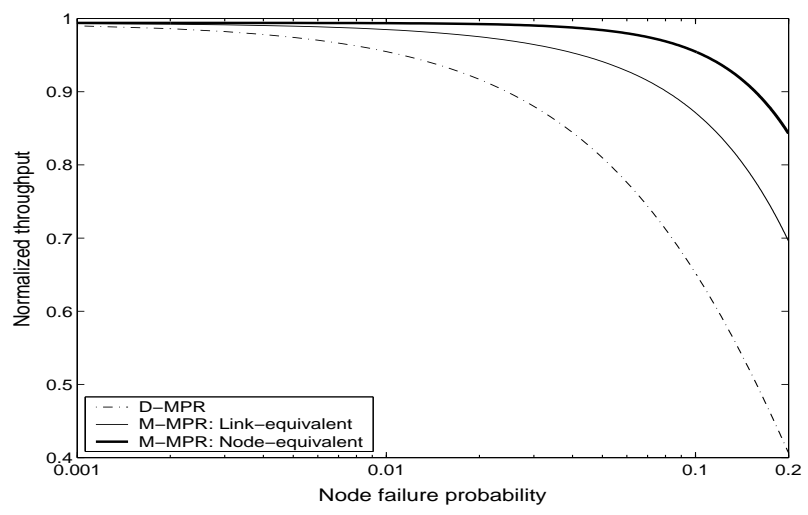

Fig. 5. Normalized throughput performance of D-MPR and equivalent M-MPR schemes. $p_{b}=10^{-6}, H=6$.

Power Consumption: Since in M-MPR with selective forwarding as well as its equivalent D-MPR schemes each node forwards the packet to only one downstream node, in an $H$-hop route total transceiver power required for each successful packet transmission is $H\left(P_{t}+P_{r}\right)$, where $P_{t}$ is the power required for a packet transmission from a node and $P_{r}$ is the corresponding receive power. However, since transmission at each node is broadcast based, all neighboring nodes along the packet forwarding route (in D-MPR as well as M-MPR) are subjected to some additional power waste. Particularly, each of these nodes have to (at least) decipher the address (header) of the packet to verify if it was directed to that node.

\section{Simulation Studies}

To verify our analytic observations we conduct Cbased discrete event simulation. We consider that the intermediate nodes may fail intermittently (with probability $p_{n}$ ). If a node is found good before transmitting a packet (based on apriori local neighborhood information), it remains good throughout the packet transmission period. However, link error can occur during a packet transmission, causing packet error. Since here we study the basic packet throughput performance, in case of packet error, no attempt is made for error correction and the corresponding packet is discarded.

The following parameter values are considered: Number of nodes 500, uniformly randomly distributed over a $500 \mathrm{~m}$ square location space; range of circular coverage of each node $40 \mathrm{~m}$; white Gaussian channel with BER $10^{-6}$, correspondingly (in Eq. 4), $K=20, C=256$, $E_{b} / N_{0}=14 d B$; packet size (fixed) 50 Bytes; number of packets per iteration (session) $10^{6}$. Sufficient number of 
sessions are simulated to achieve throughput within $95 \%$ confidence interval.

Fig. 6 shows normalized throughput plots, where the end-to-end distance is averaged from multiple sessions. We observe the similar trend of throughput performances from analysis, as shown in Fig. 5. Note that due to random placement of nodes equal length routes and idealized mesh (Figs. 2 and 3) could not be ensured. Longer multiple routes, incomplete mesh, and longer average hop length lead to poorer performances in simulated network compared to that in the analysis.

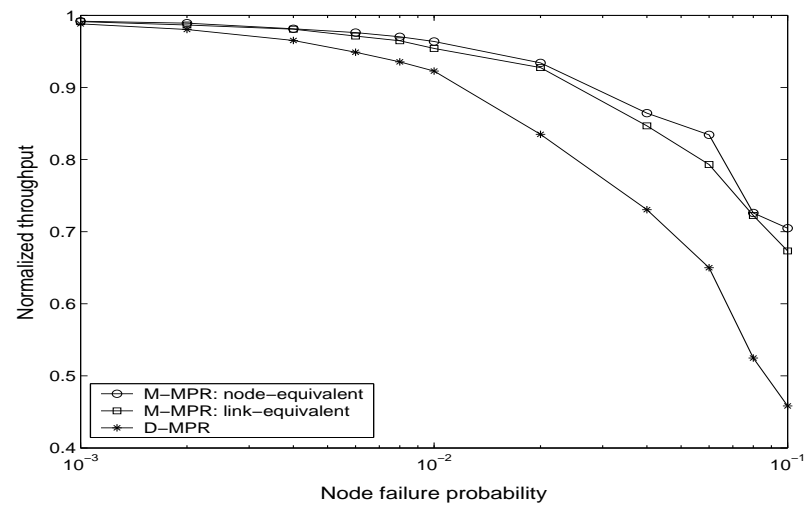

Fig. 6. Normalized throughput performance of D-MPR and its equivalent M-MPR schemes, obtained from simulations. Average number of hops 9.06 .

The variation of normalized throughput of D-MPR and its node equivalent M-MPR with end-to-end distance is shown in Fig. 7. The source-destination hop length is varied by changing the length-to-breadth ratio of location space. Observe that the throughput performance in DMPR degrades at a faster rate with respect to the MMPR. The results for receiver complexity equivalent MMPR follow closely to the node equivalent M-MPR.

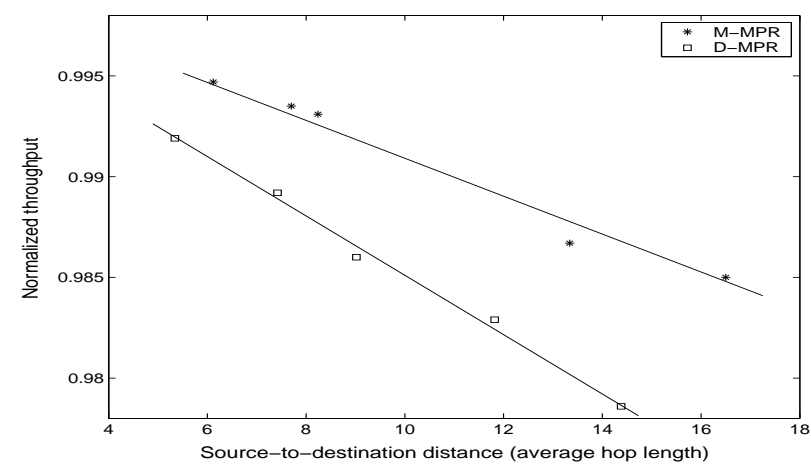

Fig. 7. Throughput variations of D-MPR and its node equivalent M-MPR with end-to-end distance. $p_{n}=10^{-3}$.

Having discussed comparative performances of D-
MPR and M-MPR, we now compare load balancing performance of M-MPR with respect to packet routing along preferential (primary) route. For fair comparison, we consider the same meshed route as in M-MPR, and the packets are forwarded along the primary route. If a failure is detected in the primary route (from local neighborhood information at each node) the packet is forwarded along a secondary route. Note that this route failure detection procedure is different from acknowledgment based rerouting [5], which incurs additional delay in failure detection and requires that the current packet be buffered at the forwarder node until the (positive) acknowledgment is received from the downstream node.

From the simulated network, an example of 4-hop source-to-destination disjoint multiple routes and its equivalent meshed routes are shown in Fig. 8.

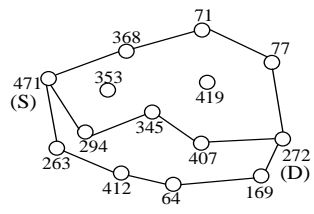

(a) disjoint multipath

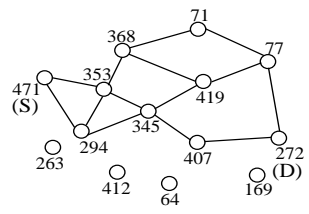

(b) meshed multipath
Fig. 8. Sketches of disjoint multipath and its node equivalent (which is also receiver complexity equivalent, in this case) meshed multipath, drawn from the network connectivity trace.

TABLE I

TRAFFIC DISTRIBUTIONS IN M-MPR AND PREFERENTIAL ROUTING IN THE MESHED ROUTE SHOWN IN FIG. 8. TOTAL NUMBER OF PACKETS $10^{6}$.

\begin{tabular}{c|c|c|l}
\hline$p_{n}$ & PLR & Routing & $\begin{array}{l}\text { Min, max \# packets } \\
\text { routed through a node }\end{array}$ \\
\hline \hline \multirow{2}{*}{$10^{-5}$} & $3.3 \times 10^{-5}$ & Preferred & 0,999978 \\
\cline { 2 - 4 } & $3.8 \times 10^{-5}$ & M-MPR & 187472,749981 \\
\hline \multirow{2}{*}{$10^{-1}$} & $4.7 \times 10^{-2}$ & Preferred & 13811,963841 \\
\cline { 2 - 4 } & $9.9 \times 10^{-2}$ & M-MPR & 181380,739646 \\
\hline
\end{tabular}

Table I shows packet loss rate (PLR $=1$-normalized throughput) and load distribution in the network with M-MPR and preferential routing, respectively, for two extreme cases of node failure rate. We note that preferential routing has a little lower PLR, as the packets mostly follow shorter routes. More specifically, at $p_{n}=10^{-5}$, observed average route length in selective forwarding based packet distribution is 4.94 hop, while that in preferential routing is 4.0 hops. At $p_{n}=10^{-1}$, the respective hop lengths are 4.43 and 4.01. However, the packet distribution in preferential routing being more uneven, it drains more battery power in certain nodes, which may eventually lead to quicker network partitioning. Moreover, since the nodes along the secondary routes are rarely used for data transmission, higher control overhead will be required (compared to M-MPR) for 
maintaining the secondary routes. We do not quantify the actual overhead for refreshing secondary routes, as this is dependent on frequency of update - a function of QoS demand of the traffic.

\section{CONCLUSion}

To summarize, we have proposed a meshed multipath routing scheme with selective packet forwarding, for wireless sensor networks, which is different from conventional acknowledgment based re-transmission and rerouting strategies. The aim has been to ensure successful data communication with minimal buffering and flow control overhead, and efficient use of network resources.

Our evaluation has shown significant improvement in throughput performance with meshed multipath over its equivalent disjoint multipath schemes, while both having the same power usage. Moreover, we have shown that with a little loss of throughput, M-MPR achieves better load balancing, which would also require lesser route maintenance overhead, with respect to packet forwarding along a preferential route.

\section{ACKNOWLEDGMENT}

The authors would like to thank Sumesh J. Philip and Jinhua Zhu for useful discussions.

\section{REFERENCES}

[1] I. F. Akyildiz, W. Su, Y. Sankarasubramaniam, and E. Cayirci, "Wireless Sensor Networks: A Survey," Computer Networks, vol. 38(4), pp. 393-422, Mar. 2002.

[2] R. V. Boppana and S. Chalasani, "Fault-Tolerant Wormhole Routing Algorithms for Mesh Networks," IEEE Trans. Computers, vol. 44(7), pp. 848-864, 1995.

[3] S. Chen and K. Nahrstedt, "Distributed Quality-of-Service Routing in Ad Hoc Networks," IEEE J. Sel. Areas on Comm., vol. 17(8), pp. 1488-1505, Aug. 1999.

[4] P. Enge and P. Misra, Eds., "Spl. Issue on GPS: The Global Positioning System," Proc. of the IEEE, pp. 3-172, Jan. 1999.

[5] D. Ganesan, R. Govindan, S. Shenker, and D. Estrin, "Highly-Resilient, Energy-Efficient Multipath Routing in Wireless Sensor Networks," ACM Mobile Computing and Communications Review, vol. 5(4), Oct. 2001.

[6] C. Intanagonwiwat, R. Govindan, and D. Estrin, "Directed Diffusion: A Scalable and Robust Communication Paradigm for Sensor Networks," in Proc. MOBICOM, 2000.

[7] P. Kermani and L. Kleinrock, "Virtual Cut-Through: A New Communication Switching Technique," J. Computer Networks, vol. 3(11), pp. 267-286, 1979.

[8] S.-J. Lee and M. Gerla, "Split Multipath Routing with Maximally Disjoint Paths in Ad hoc Networks," in Proc. Intl. Conf. on Comm. (ICC), 2001.

[9] A. Nasipuri and S. R. Das, "On-Demand Multipath Routing for Mobile Ad Hoc Networks," in Proc. Intl. Conf. on Computer Comm. and Networks (IC3N), pp. 64-70, Oct. 1999.

[10] T. Rappaport, Wireless Communications: Principles and Practice. Prentice Hall, 1996.

[11] A. Tsirigos and Z. J. Hass, "Multipath Routing in The Presence of Frequent Topological Changes," IEEE Comm. Magazine, pp. 132-138, Nov. 2001.

[12] F. Ye, S. Lu, and L. Zhang, "GRAdient Broadcast: A Robust, Long-Lived Large Sensor Network," in UCLA CS Tech. Report, Sep. 2001.

\section{APPENDIX}

\section{Recursive Calculation of $P_{s}(2)$ IN M-MPR}

$P_{\left\lfloor\frac{H}{2}\right\rfloor, i+1}, 0 \leq i \leq\left\lfloor\frac{H}{2}\right\rfloor$, is obtained from Eq. (5).

BEGIN

$$
\begin{aligned}
& \text { IF } H \text { odd, } \\
& \text { FOR } j=1 \text { through }\left\lfloor\frac{H}{2}\right\rfloor \text {, with increment } 2, \\
& \quad P_{\left\lceil\frac{H}{2}\right\rceil, j} \leftarrow \frac{P_{\left\lfloor\frac{H}{2}\right\rfloor, j}+P_{\left\lfloor\frac{H}{2}\right\rfloor, j+1}}{2}\left(1-p_{n}^{2}\right)\left(1-p_{l}\right) \\
& \quad P_{\left\lceil\frac{H}{2}\right\rceil, j+1} \leftarrow P_{\left\lceil\frac{H}{2}\right\rceil, j} \\
& \text { end FOR } \\
& \quad \text { IF }\left\lfloor\frac{H}{2}\right\rfloor \text { even, } \\
& \quad P_{\left\lceil\frac{H}{2}\right\rceil,\left\lceil\frac{H}{2}\right\rceil} \leftarrow P_{\left\lfloor\frac{H}{2}\right\rfloor,\left\lceil\frac{H}{2}\right\rceil}\left(1-p_{n}\right)\left(1-p_{l}\right) \\
& \text { end IF } \\
& \text { end IF } \\
& \text { FOR } i=\left\lceil\frac{H}{2}\right\rceil+1 \text { through } H-1, \text { with increment } 1, \\
& \quad P_{i, 1} \leftarrow P_{i-1,1}\left(1-p_{n}\right)\left(1-p_{l}\right)+\frac{P_{i-1,2}}{2}\left(1-p_{n}^{2}\right)\left(1-p_{l}\right) \\
& \quad j \leftarrow H+1-i \\
& P_{i, j} \leftarrow \frac{P_{i-1, j}}{2}\left(1-p_{n}^{2}\right)\left(1-p_{l}\right)+P_{i-1, j+1}\left(1-p_{n}\right)\left(1-p_{l}\right) \\
& \text { FOR } j=2 \text { through } H-i, \text { with increment } 1, \\
& \quad P_{i j} \leftarrow \frac{P_{i-1, j}+P_{i-1, j+1}}{2}\left(1-p_{n}^{2}\right)\left(1-p_{l}\right) \\
& \text { end FOR } \\
& \text { end FOR } \\
& P_{s}(2)=P_{H-1,1}+P_{H-1,2}
\end{aligned}
$$

END 